\title{
A Cost-Sensitive Paradigm for Multiclass to Binary Decomposition Schemes
}

\author{
Claudio Marrocco and Francesco Tortorella \\ Dipartimento di Automazione, Elettromagnetismo \\ Ingegneria dell'Informazione e Matematica Industriale \\ Università degli Studi di Cassino \\ 03043 Cassino (FR), Italy \\ \{marrocco, tortorella\}@unicas.it
}

\begin{abstract}
An established technique to face a multiclass categorization problem is to reduce it into a set of two-class problems. To this aim, the main decomposition schemes employed are one vs. one, one vs. all and Error Correcting Output Coding. A point not yet considered in the research is how to apply these methods to a cost-sensitive classification that represents a significant aspect in many real problems. In this paper we propose a novel method which, starting from the cost matrix for the multi-class problem and from the code matrix employed, extracts a cost matrix for each of the binary subproblems induced by the coding matrix. In this way, it is possible to tune the single two-class classifier according to the cost matrix obtained and achieve an output from all the dichotomizers which takes into account the requirements of the original multi-class cost matrix. To evaluate the effectiveness of the method, a large number of tests has been performed on real data sets. The experiments results have shown a significant improvement in terms of classification cost, specially when using the ECOC scheme.
\end{abstract}

\section{Introduction}

A diffused technique to face a classification problem with many possible classes is to decompose the original problem into a set of two-class problems. The rationale of this approach rely on the stronger theoretical roots and better comprehension characterizing two class classifiers (dichotomizers) such as Perceptrons or Support Vector Machines. Moreover, with this method it becomes possible to employ in multi class problems some dichotomizers which are very effective in two-class problems but are not capable to directly perform multi-class classification. To this aim, the main decomposition schemes employed are one vs. one, one vs. all and Error Correcting Output Coding. However, a point not yet considered is how to devise a decomposition scheme for cost-sensitive classification. This is a significant point in many real problems such as automated disease diagnosis, currency recognition, speaker identification, and fraud detection in which different classification errors frequently have consequences of very different gravity. For this reason, the classification systems

\footnotetext{
A. Fred et al. (Eds.): SSPR\&SPR 2004, LNCS 3138, pp. 753-761, 2004.

(C) Springer-Verlag Berlin Heidelberg 2004
} 
used in such situations must take into account the different costs and benefits (collected in a cost matrix) which the different decisions can provide and thus should be tuned accordingly. In mult-class classifiers, such task is usually accomplished by modifying the learning algorithm used during the training phase of the classifier or by tuning the classifier after the learning phase.

In this paper we propose a cost-sensitive paradigm for multiclass to binary decomposition schemes. Starting from the cost matrix for the multi-class problem and from the code matrix employed, a cost matrix is derived for each of the binary problems induced by the columns of the code matrix. In this way it is possible to tune the single dichotomizer according to the cost matrix obtained and achieve an output from the dichotomizers which takes into account the requirements of the original multi-class cost matrix.

In the rest of the paper we describe, after a short description of the main decomposition schemes, how to decompose the original multi-class cost matrix in more two-class cost matrices. A conclusive section describes the results obtained from experiments performed on real data set.

\section{Output Coding for Multi-class Problems}

In order to introduce the main decomposition methods, let us consider a problem with $n$ classes to be reduced to a set of $L$ binary problems. For each problem, we employ a dichotomizer, i.e. a classifier able to discriminate between two mutually exclusive classes that can be generically called Positive $(P)$ class (labelled by +1$)$ and Negative $(N)$ class (labelled by -1 ).

The most immediate approach is to create one binary problem for each class $i$, in which all samples labelled $i$ are considered positive samples while all other samples are considered negative. Such method is frequently defined one vs. all (OVA) and involves the definition of $L=n$ binary problems. Another approach, suggested by Hastie and Tibshirani [1], is to define as many binary problems as the possible pairs of different classes. For a given pair of distinct classes $\{i, j\}$, the corresponding binary problem considers positive the samples belonging to $i$ and negative the samples belonging to $j$; all other samples are ignored. This is the one vs. one (OVO) approach; in this case, we have to define $L=n(n-1) / 2$ binary problems.

A further technique that has emerged for its good generalization capabilities is the Error Correcting Output Coding (ECOC), introduced by Dietterich and Bakiri in [2], which is based on a $n \times L$ coding matrix $M=\left\{M_{h k}\right\}$, where $M_{h k}= \pm 1$. Each row of $M$ corresponds to a bit string, called codeword, that represents a class label, while each column corresponds to a binary problem. Usually, it is chosen $L>n$, so as to make the Hamming distance between every pair of strings as large as possible. In this way, if $d_{m}$ is the minimum Hamming distance between any pair of codewords, the code is able to correct at least $\left\lfloor\left(d_{m}-1\right) / 2\right\rfloor$ single bit errors. 
The coding matrix can be used to model also the OVA approach: this kind of decomposition, in fact, is represented by a square coding matrix $M$ in which $M_{k k}=+1$ and $M_{h k}=-1 \forall h \neq k$. Moreover, we can generalize the coding matrix as suggested in [3] and assume that $M_{h k}$ can be also 0 , thus indicating that the $k$-th binary problem does not consider samples of the class $h$. In this way, the OVO approach can be represented by a $n \times n(n-1) / 2$ coding matrix. In such case, if we suppose that the $k$-th dichotomizer must decide between class $i$ and class $j$, we will have $M_{i k}=+1, M_{j k}=-1$ (or vice versa) and $M_{h k}=0, \forall h \neq i, j$.

It is worth noting that both OVA and OVO decomposition schemes do not provide the same robustness given by the ECOC to errors made by the dichotomizers. In fact, while the ECOC matrix is built so as to guarantee a large value of distance between different codewords, the OVA scheme entails a fixed distance of 2 between different codewords. The situation is even worse for the OVO scheme for which the distance between distinct codewords is unitary because the 0 values in the matrix act as don't care and thus are not considered in the evaluation of the distance.

Once the coding matrix has been defined and the dichotomizers have been trained, to classify a new sample $x$, a vector of binary decisions is computed by applying each of the learned dichotomizers to $x$; to decode the resulting vector, i.e. to pass from the binary to the multi-class problem, the most common approach consists in evaluating the Hamming distances between the vector and the codewords of the matrix and choose for the nearest code word, i.e. for the minimum Hamming distance. Other decoding rules have been proposed which are based on a Least Squares approach [4] or on the loss function employed in the training algorithm of the dichotomizer [3], but we will not consider them in this paper.

\section{Evaluating the Binary Costs from the Multi-class Costs}

Before analyzing cost-sensitive classification in the multi-class case, it is convenient to focus preliminarily on the two-class problem. In this case, a sample can be assigned to one of two mutually exclusive classes that we have defined $\mathrm{n}$ the previous section as Positive class and Negative class. The set of samples classified as "positive" by the dichotomizer will contain some actually-positive samples correctly classified and some actually-negative samples incorrectly classified. Hence, two appropriate performance figures are given by the True Positive Rate (TPR), i.e. the fraction of actually-positive cases correctly classified, and by the False Positive Rate (FPR), given by the fraction of actually-negative cases incorrectly classified as "positive". In a similar way, it is possible to evaluate the True Negative Rate (TNR) and the False Negative Rate $(F N R)$. It is worth noting that only two indices are actually necessary because the following relations hold:

$$
F N R=1-T P R \quad T N R=1-F P R .
$$

In cost sensitive applications, every decision taken by the classifier involves a cost which estimates the penalty (benefit) produced by an error (by a correct decision). In 
many applications the two kinds of error (false positive and false negative) are not equally costly as well as the value of the benefit obtained can depend on the class of the sample correctly identified. Hence, we have to consider a cost matrix similar to the one described in table 1. It is worth noting that, while $C F N$ (Cost for a False Negative) and CFP (Cost for a False Positive) have positive values, CTP (Cost for a True Positive) and CTN (Cost for a True Negative) are negative costs since they actually represent a benefit.

Table 1. Cost matrix for a two class problem.

\begin{tabular}{c|c|c|c|}
\multicolumn{1}{c}{} & \multicolumn{3}{c}{ True class } \\
\cline { 2 - 4 } \multicolumn{1}{c}{} & $N$ & $P$ \\
Predicted & $N$ & $C T N$ & $C F N$ \\
\cline { 2 - 4 } Class & $P$ & $C F P$ & $C T P$ \\
\cline { 2 - 4 } & & &
\end{tabular}

With such assumptions an estimate of the effectiveness of a dichotomizer working in a cost sensitive application can be given by the expected classification cost (EC) defined as:

$$
\begin{aligned}
E C= & p(P) \cdot C F N \cdot F N R+p(N) \cdot C F P \cdot F P R+ \\
& p(P) \cdot C T P \cdot T P R+p(N) \cdot C T N \cdot T N R
\end{aligned}
$$

where $p(P)$ and $p(N)$ are the a priori probabilities of the positive and negative classes.

It is easy to show [5] that the cost matrix in table 1 is equivalent to the cost matrix in table 2 which depends only on the cost ratio $\rho=\frac{C F N-C T P}{C F P-C T N}$.

Table 2. Cost matrix for a two class problem.

\begin{tabular}{c|c|c|c|}
\cline { 3 - 4 } \multicolumn{1}{c|}{} & \multicolumn{3}{c}{ True class } \\
\cline { 2 - 4 } \multicolumn{1}{c}{} & $N$ & $P$ \\
\hline \multirow{3}{*}{$\begin{array}{c}\text { Predicted } \\
\text { Class }\end{array}$} & $N$ & 0 & $\frac{C F N-C T P}{C F P-C T N}$ \\
\cline { 2 - 4 } & $P$ & 1 & 0 \\
\cline { 2 - 4 } & &
\end{tabular}

As a consequence, the expressions of the expected cost changes in:

$$
E C=p(N) \cdot F P R+p(P) \cdot F N R \cdot \rho
$$

Let us now consider a multi class problem with $n$ classes to be reduced to $L$ binary problems by using a $n \times L$ coding matrix $M=\left\{M_{h k}\right\}$ where $M_{h^{*}}$ is the codeword for the class $h$ and $M_{h k}$ is the label assumed by a sample belonging to the class $h$ in the binary problem induced by the $k$-th column. Moreover, let us assume that the costs of the multi-class problems are described by a $n \times n$ cost matrix $C=\left\{C_{i j}\right\}$ where $C_{i j}>0$ represents the cost produced by assigning to the class $j$ a sample actually belonging to the class $i$; the cost for a correct classification is null, i.e. $C_{i i}=0 \forall i$. 
Let us define $N^{(k)}=\left\{h \mid M_{h k}=-1\right\}$ the set of classes labelled with -1 and $P^{(k)}=\left\{h \mid M_{h k}=+1\right\}$ the set of classes labelled with +1 in the $k$-th binary problem. In an analogous way, let us call $C_{F P}^{(k)}$ and $C_{F N}^{(k)}$ the cost produced in the operative phase by the dichotomizer trained on the $k$-th problem when it erroneously assigns to $P^{(k)}$ a sample belonging to $N^{(k)}$ and vice versa.

To establish the values of $C_{F P}^{(k)}$ and $C_{F N}^{(k)}$, let us consider which are the consequences on the multi-class problem of an error made by the $k$-th dichotomizer. A false positive error moves one unit away from the true codewords containing a -1 in the $k$-th position toward the erroneous codewords containing a +1 in the same position. In particular, if $r$ and $s$ are two classes such that $r \in N^{(k)}$ and $s \in P^{(k)}$, a false positive error made by the $k$-th dichotomizer on a sample belonging to $r$ will move one unit from the correct codeword of $r, M_{r^{*}}$, toward the codeword of $s, M_{s^{*}}$. Let us call $\mathrm{d}\left(M_{r^{*}}, M_{s^{*}}\right)$ the Hamming distance existing between $M_{r^{*}}$ and $M_{s^{*}}$; if there were errors also on the other $\mathrm{d}\left(M_{r^{*}}, M_{s^{*}}\right)$ bits in which the two codewords differ, an error (with a cost equal to $C_{r s}$ ) would be generated in the multi-class problem. The contribution to such error given by the false positive produced by the $k$-th dichotomizer can be hence estimated as $\frac{1}{\mathrm{~d}\left(M_{r^{*}}, M_{s^{*}}\right)}$; as a consequence, the cost of the false positive related to the possible misclassification between $r$ and $s$ can be estimated as $\frac{C_{r s}}{\mathrm{~d}\left(M_{r^{*}}, M_{s^{*}}\right)}$.

Actually, the false positive moves the $M_{r^{*}}$ toward all the codewords belonging to $P^{(k)}$ and thus the cost related to all the possible misclassifications involving the class $r$ can be estimated as:

$$
\sum_{s \in P^{(k)}} \frac{C_{r s}}{\mathrm{~d}\left(M_{r^{*}}, M_{s^{*}}\right)}
$$

Eventually, we have to extend such evaluation to all the classes belonging to $N^{(k)}$. The conclusion is that the cost for a false positive made by the $k$-th dichotomizer is related to the risk of misclassifying one of the classes belonging to $N^{(k)}$ with one from $P^{(k)}$ and an estimate of its value is:

$$
C_{F P}^{(k)}=\sum_{r \in N^{(k)}} \sum_{s \in P^{(k)}} \frac{C_{r s}}{\mathrm{~d}\left(M_{r^{*}}, M_{s^{*}}\right)}
$$

Likewise, it is possible to estimate the cost for a false negative made by the $k$-th dichotomizer since it is related to the risk of misclassifying one of the classes belonging to $P^{(k)}$ with one from $N^{(k)}$ :

$$
C_{F N}^{(k)}=\sum_{u \in P^{(k)}} \sum_{v \in N^{(k)}} \frac{C_{u v}}{\mathrm{~d}\left(M_{u^{*}}, M_{v^{*}}\right)}
$$

In this way, we can define for the $k$-th dichotomizer a cost matrix similar to the one shown in table 2 with cost ratio: 


$$
\rho_{E C O C}^{(k)}=\frac{\sum_{u \in P^{(k)}} \sum_{v \in N^{(k)}} \frac{C_{u v}}{\mathrm{~d}\left(M_{u^{*}}, M_{v^{*}}\right)}}{\sum_{r \in N^{(k)}} \sum_{s \in P^{(k)}} \frac{C_{r s}}{\mathrm{~d}\left(M_{r^{*}}, M_{s^{*}}\right)}}
$$

It is easy to see that the conditions for a realistic cost matrix (i.e. $\left.0<\rho^{(k)}<+\infty\right)$ are satisfied since $C_{i j}>0 \forall i \neq j$ and $\mathrm{d}\left(M_{r^{*}}, M_{s^{*}}\right) \neq 0 \forall r \neq s$.

Taking into account the generalized form of the coding matrix introduced in [3], eq. (7) is valid for each of the decomposition methods previously described. However it takes simpler expressions when a OVA or a OVO method is used. In the first case the expressions for $C_{F P}^{(k)}$ and $C_{F N}^{(k)}$ become:

$$
C_{F P}^{(k)}=\sum_{r \neq k} \frac{C_{r k}}{\mathrm{~d}\left(M_{r^{*}}, M_{k^{*}}\right)}=\frac{1}{2} \sum_{r \neq k} C_{r k} \quad C_{F N}^{(k)}=\sum_{v \neq k} \frac{C_{k v}}{\mathrm{~d}\left(M_{k^{*}}, M_{v^{*}}\right)}=\frac{1}{2} \sum_{v \neq k} C_{k v}
$$

while the corresponding cost ratio is:

$$
\rho_{\text {OVA }}^{(k)}=\frac{\sum_{v \neq k} C_{k v}}{\sum_{r \neq k} C_{r k}}
$$

In the same way, it is possible to obtain the expressions of the costs for the binary problems induced by the OVO decomposition scheme. Let us suppose, without loss of generalization, that $M_{i k}=+1, M_{j k}=-1$ and $M_{h k}=0, \forall h \neq i, j$, i.e. that the $k$-th dichotomizer must decide between class $i$ and class $j$, with $P^{(k)}=\{i\}$ and $N^{(k)}=\{j\}$. Taking into account that $\mathrm{d}\left(M_{i^{*}}, M_{j^{*}}\right)=1 \forall i \neq j$, the corresponding costs are:

$$
C_{F P}^{(k)}=\frac{C_{j i}}{\mathrm{~d}\left(M_{j^{*}}, M_{i^{*}}\right)}=C_{j i} \quad C_{F N}^{(k)}=\frac{C_{i j}}{\mathrm{~d}\left(M_{i^{*}}, M_{j^{*}}\right)}=C_{i j}
$$

while the cost ratio is:

$$
\rho_{O V O}^{(k)}=\frac{C_{i j}}{C_{j i}}
$$

\section{Experimental Results}

To evaluate the effectiveness of the proposed approach we have made several experiments on different data sets and compared the classification costs obtained with the different decomposition schemes. To this aim, a comparison technique has been devised to assure that the outcomes obtained were statistically significant.

The data sets used are publicly available at the UCI Machine Learning Repository [6]; all of them have numerical input features and a variable number of classes. The features were previously rescaled so as to have zero mean and unit standard deviation. More details of data sets are given in table 3 . The table provides also the type of ECOC coding matrix used for each data set. We choose an exhaustive code [2] for 
the sets that have a number of classes lower than 8 and a $\mathrm{BCH}$ code [2] for those having a number of classes greater or equal to 8. In particular, for Vowel set we adopted ECOC codes 15-11 available at http://web.engr.oregonstate.edu/ tgd.

Table 3. Data sets and coding matrices used in the experiments.

\begin{tabular}{|c|c|c|c|c|c|c|c|c|}
\hline data Set & classes & feat. & samples & $\begin{array}{c}\text { train. } \\
\text { set }\end{array}$ & $\begin{array}{c}\text { test } \\
\text { set }\end{array}$ & $\begin{array}{c}\text { valid. } \\
\text { set }\end{array}$ & $\begin{array}{c}\text { ECOC } \\
\text { matrices }\end{array}$ & $\begin{array}{c}\text { ECOC } \\
\text { length }\end{array}$ \\
\hline Ann-thyroid & 3 & 21 & 7200 & 5040 & 1080 & 1080 & Exhaustive & 3 \\
\hline Dermatology. & 6 & 34 & 358 & 252 & 54 & 52 & Exhaustive & 31 \\
\hline Glass & 6 & 9 & 214 & 149 & 32 & 33 & Exhaustive & 31 \\
\hline Sat Image & 6 & 36 & 6435 & 4505 & 965 & 965 & Exhaustive & 31 \\
\hline Segmentation & 7 & 18 & 2310 & 1617 & 350 & 343 & Exhaustive & 63 \\
\hline Optdigits & 10 & 62 & 5620 & 3935 & 844 & 841 & BCH 31-21 & 31 \\
\hline Pendigits & 10 & 16 & 10992 & 7696 & 1647 & 1649 & BCH 31-21 & 31 \\
\hline Vowel & 11 & 10 & 990 & 693 & 154 & 143 & Diett 15-11 & 15 \\
\hline
\end{tabular}

The dichotomizer employed is a Support Vector Machine with a RBF kernel; it has been implemented by SVM ${ }^{\text {light }}$ tool [7] available at http://svmlight.joachims.org. In order to build dichotomizers tuned on the cost matrices determined according the method described in Section 3, we have adopted a post-learning scheme [5] which evaluates a threshold $t$ to be imposed on the output of the SVM, so as to attribute the sample to be classified to the class $N$ if the SVM output is less than $t$ and to the class $P$ otherwise. The threshold is chosen so as to minimize the expected classification cost on a validation set.

We have compared each other the different results obtained with the decomposition schemes previously described. In particular, we have considered both standard versions of the ECOC (E-N), of the OVA (A-N) and of the OVO $(\mathrm{O}-\mathrm{N})$ which use dichotomizers without any cost-sensitive tuning and the corresponding cost-sensitive versions (E-C, A-C, O-C), where the dichotomizers are tuned according to the cost matrices built as seen in Section 3.

To avoid any bias in the comparison, 12 runs of a multiple hold-out procedure were performed on all data sets. In each run, the data set was split in three subsets: a training set (containing $70 \%$ of the samples of each class), a validation set and a test set (each containing $15 \%$ of the samples of each class); the final size of each of these sets is given in table 3 . The validation set is used to evaluate the optimal threshold for the cost-sensitive tuning, while it is considered as part of the training set for the not cost-sensitive tuned dichotomizers.

The classification costs to be compared were evaluated on the test set, thus obtaining, for a given data set, 12 different values for each of the costs required. To establish a statistically significant comparison among all the decomposition schemes (both standard and cost-sensitive), we have used the Tukey's method [8] that tests all possible pairwise differences of means of distinct populations and determines if each difference is significantly lower than, higher than or undistinguishable from 0 . All the results are provided with a level of significance equal to 0.05 . To obtain a result unbiased with respect to the particular cost values, we apply the approach proposed in [9]: a hundred of different cost matrices have been used whose elements were randomly 
generated according to a uniform distribution over the range $[1,10]$. For each cost matrix, the test before described has been repeated.

In tables 4-11 the results of the comparisons performed are presented. We have a table in which the element $T(p, q)$ on row $p$ and column $q$ indicates the number of runs (out of 100) for which the $p$-th scheme has produced a cost higher than the $q$-th scheme. For example, the element $T(2,4)$ indicates the number of runs in which the A-N scheme gives a classification cost higher than the E-C scheme, while the symmetrical element $T(4,2)$ reports the number of runs in which the $\mathrm{E}-\mathrm{C}$ gives a higher cost. The number of runs in which the $p$-th and the $q$-th schemes provide costs not significantly different is given by $100-T(p, q)-T(q, p)$.

Table 4. Results for ANN Thyroid data set.

\begin{tabular}{rrrrr|r|r|r|} 
& E-N & A-N & O-N & E-C & A-C & O-C \\
E-N & 0 & 0 & 0 & 100 & 100 & 100 \\
A-N & 0 & 0 & 0 & 100 & 100 & 100 \\
O-N & 0 & 0 & 0 & 100 & 100 & 100 \\
\hline E-C & 0 & 0 & 0 & 0 & 0 & 26 \\
A-C & 0 & 0 & 0 & 0 & 0 & 27 \\
O-C & 0 & 0 & 0 & 0 & 0 & 0 \\
\hline
\end{tabular}

Table 6. Results for Glass data set.

\begin{tabular}{l|r|r|r|r|r|r|} 
& E-N & A-N & O-N & E-C & A-C & O-C \\
E-N & 0 & 5 & 0 & 68 & 37 & 62 \\
A-N & 0 & 0 & 0 & 63 & 17 & 50 \\
O-N & 28 & 57 & 0 & 84 & 60 & 77 \\
\hline E-C & 0 & 0 & 0 & 0 & 0 & 0 \\
A-C & 10 & 6 & 6 & 45 & 0 & 37 \\
O-C & 0 & 0 & 0 & 0 & 1 & 0 \\
\hline
\end{tabular}

Table 8. Results for Segmentation data set.

\begin{tabular}{|r|r|r|r|r|r|r|} 
& E-N & A-N & O-N & E-C & A-C & O-C \\
\hline E-N & 0 & 7 & 22 & 80 & 34 & 0 \\
A-N & 65 & 0 & 67 & 92 & 78 & 64 \\
\hline O-N & 33 & 9 & 0 & 69 & 35 & 21 \\
\hline E-C & 0 & 0 & 0 & 0 & 8 & 0 \\
A-C & 31 & 0 & 29 & 58 & 0 & 25 \\
\hline O-C & 5 & 8 & 21 & 85 & 32 & 0 \\
\hline
\end{tabular}

Table 10. Results for Pendigits data set.

\begin{tabular}{l|r|r|r|r|r|r|} 
& E-N & A-N & O-N & E-C & A-C & O-C \\
\hline E-N & 0 & 15 & 0 & 44 & 16 & 37 \\
A-N & 63 & 0 & 58 & 70 & 0 & 59 \\
\hline O-N & 3 & 17 & 0 & 49 & 18 & 40 \\
\hline E-C & 12 & 8 & 6 & 0 & 7 & 3 \\
A-C & 61 & 0 & 56 & 66 & 0 & 56 \\
\hline O-C & 35 & 13 & 30 & 41 & 15 & 0 \\
\hline
\end{tabular}

Table 5. Results for Dermatology data set.

\begin{tabular}{rr|r|r|r|r|r|}
\multicolumn{1}{l|}{} & E-N & A-N & O-N & E-C & A-C & O-C \\
E-N & 0 & 0 & 0 & 100 & 100 & 96 \\
A-N & 0 & 0 & 0 & 100 & 100 & 96 \\
O-N & 0 & 0 & 0 & 100 & 100 & 97 \\
\hline E-C & 0 & 0 & 0 & 0 & 0 & 8 \\
\hline A-C & 0 & 0 & 0 & 52 & 0 & 40 \\
O-C & 0 & 0 & 0 & 30 & 8 & 0 \\
\hline
\end{tabular}

Table 7. Results for Satimage data set.

\begin{tabular}{r|r|r|r|r|r|r|} 
& E-N & A-N & O-N & E-C & A-C & O-C \\
\hline E-N & 0 & 0 & 0 & 60 & 30 & 85 \\
A-N & 0 & 0 & 0 & 60 & 30 & 85 \\
\hline O-N & 0 & 0 & 0 & 69 & 33 & 90 \\
\hline E-C & 2 & 2 & 0 & 0 & 2 & 54 \\
\hline A-C & 30 & 30 & 29 & 51 & 0 & 77 \\
\hline O-C & 0 & 0 & 0 & 1 & 1 & 0 \\
\hline
\end{tabular}

Table 9. Results for OptdiEgits data set.

\begin{tabular}{|r|r|r|r|r|r|r|} 
& E-N & A-N & O-N & E-C & A-C & O-C \\
\hline E-N & 0 & 27 & 21 & 91 & 72 & 84 \\
A-N & 28 & 0 & 3 & 98 & 86 & 93 \\
\hline O-N & 31 & 9 & 0 & 98 & 87 & 97 \\
\hline E-C & 0 & 0 & 0 & 0 & 0 & 0 \\
A-C & 2 & 0 & 0 & 47 & 0 & 39 \\
\hline O-C & 0 & 0 & 0 & 0 & 3 & 0 \\
\hline
\end{tabular}

Table 11. Results for Vowel data set.

\begin{tabular}{|r|r|r|r|r|r|r|r|} 
& E-N & A-N & O-N & E-C & A-C & O-C \\
\hline E-N & 0 & 13 & 10 & 19 & 13 & 8 \\
A-N & 60 & 0 & 43 & 63 & 0 & 36 \\
\hline O-N & 41 & 9 & 0 & 23 & 9 & 2 \\
\hline E-C & 30 & 13 & 0 & 0 & 13 & 0 \\
\hline A-C & 60 & 0 & 45 & 64 & 0 & 36 \\
\hline O-C & 58 & 28 & 32 & 54 & 27 & 0 \\
\hline
\end{tabular}


It is worth noting that each table can be divided in four quadrants (evidenced by thick lines), each corresponding to a distinct kind of comparison: in particular, the top right (bottom left) quadrant reports the number of runs in which a cost-sensitive scheme works better (worse) than a non cost-sensitive scheme, while the top left (bottom right) quadrant contains the results of comparisons among the non cost-sensitive (cost-sensitive) schemes.

Let us firstly compare the cost-sensitive and non cost-sensitive versions of the decomposition schemes. We can note that cost-sensitive ECOC gives always better results than its non cost-sensitive counterpart except for Vowel data set. A similar behavior is shown by the OVA scheme, even though there are four cases (Glass, Satimage, Pendigits and Vowel) in which the two versions of the scheme are equivalent and by the OVO scheme that is equivalent to the standard version on Segmentation and Pendigits while performs significantly worse on Vowel data set.

If we focus only on cost-sensitive schemes, it is evident the superiority of the ECOC scheme with respect to OVA in all the cases except the ANN-Thyroid set, where the two schemes are equivalent. On the other side, the comparison between ECOC and OVO shows that the first scheme is better in half of the considered sets, while OVO outperforms ECOC in the ANN-Thyroid and Satimage sets. ECOC and OVO appear to be equivalent in Glass and Optdigits sets.

In summary, the experiments show that the proposed method achieves an improvement in terms of classification cost in cost-sensitive applications. This is specially true when using an ECOC decomposition scheme, which exploits its major robustness with respect to OVA and OVO schemes.

\section{References}

1. Hastie, T., Tibshirani, R.: Classification by Pairwise Coupling. The Annals of Statistics 26 (1998) 451-471

2. Dietterich, T.G., Bakiri, G.: Solving Multiclass Learning Problems via Error Correcting Output Codes. Journal of Artificial Intelligence Research 2 (1995) 263-286

3. Allwein, E.L., Schapire, R.E., Singer, Y.: Reducing Multiclass to Binary: A Unifying Approach for Margin Classifiers. Journal of Machine Learning Research 1 (2000) 113-141

4. Windeatt, T., Ghaderi, R.: Coding and Decoding Strategies for Multi Class Learning Problems. Information Fusion 4 (2003) 11-21

5. Tortorella, F.: An Empirical Comparison of In-Learning and Post-Learning Optimization Schemes for Tuning the Support Vector Machines in Cost-Sensitive Applications. Proc. 12th Int. Conf. on Image Anal. and Proc., IEEE Computer Society Press (2003), 560-565

6. Blake, C., Keogh, E., Merz, C.J.: UCI Repository of Machine Learning Databases. (1998) [www.ics.uci.edu/ mlearn/MLRepository.html]

7. Joachims, T.: Making Large-Scale SVM Learning Practical, in Schölkopf, B., Burges, C.J.C., Smola, A.J., eds., Advances in Kernel Methods, MIT Press (1999) 169-184

8. NIST/SEMATECH e-Handbook of Statistical Methods. http://www.itl.nist.gov/div898/handbook/ (2003)

9. Margineantu, D.D., Dietterich, T.G., Bootstrap Methods for the Cost-Sensitive Evaluation of Classifiers. Proc. Int. Conf. Machine Learning ICML-2000 (2000), 582-590 